\title{
Diagnosis of a Deep-Seated Intermuscular Angiolipoma Between the Trapezius and Rhomboid Major Muscles
}

\section{Adesanya Olamide Adewale}

Kampala International University - Western Campus

Olatayo Segun Okeniran

Kampala International University - Western Campus

Fred Ssempijja ( $\sim$ kalanzifr@yahoo.com )

Kampala International University - Western Campus https://orcid.org/0000-0003-1849-7185

Adam Moyosore Afodun

Kampala International University - Western Campus

Ojewale Abdulfatai Olakunle

Kampala International University - Western Campus

John Tabakwot Ayuba

Kampala International University - Western Campus

Theophilus Pius

Kampala International University - Western Campus

Amnia Diaz Anaya

Kampala International University - Western Campus

\section{Research article}

Keywords: Angiolipoma, Axioappendicular, Cadaver, Intermuscular, Trapezius muscle, Rhomboid major, Dissection

Posted Date: September 10th, 2020

DOI: https://doi.org/10.21203/rs.3.rs-66912/v1

License: (c) (i) This work is licensed under a Creative Commons Attribution 4.0 International License. Read Full License 


\section{Abstract}

Background: Lipomas are benign tumors contributing $1 \%$ of tumors, and $50 \%$ of soft tissue tumors. They are usually in subcutaneous tissues, deeply-seated lipomas are rare. To our knowledge, this is one of the few cases of intermuscular lipomas around East Africa. The purpose of this study was to describe the gross and microscopic features of a deep-seated lipoma and its clinical anatomy in the context of a live person.

Method: This was an experimental qualitative and quantitative study on specimen from an adult cadaver at the Laboratory of the Department of Human Anatomy of Kampala International University, Western Campus. The lipoma was examined in situ, then excised, measured, weighed, then routine histological tissue processing and examination done.

Results: A capsulated homogenous yellow mass was located between the trapezius muscle above and the rhomboid major muscle below, with its prominent blood supply as a continuation of the dorsal scapular blood vessels from the rhomboid. Its weight was $47.03 \mathrm{~g}$, with $8.5 \mathrm{~cm} \times 6.6 \mathrm{~cm} \times 1.7 \mathrm{~cm}$ (length, width, and thickness). Microscopic analysis shows mature fat cells and prominent blood vessels (angiogenic blood invasion).

Conclusions: This is one of the few cases of intermuscular angiolipomas, may cause severe clinical features due to vessel impingement to adjourning soft tissues, muscles, and joints if the growth continued undiscovered, unabated and treatment not offered on time. Further studies are recommended to establish the aspects of medical, treatment/interventions, family, and psychosocial history, and outcome regarding intermuscular angiolipomas in patients.

\section{Background}

Lipomas are benign mesenchymal tumors of fat cells and are the most common form of soft tissue benign tumors (account for almost $50 \%$ of all soft tissue tumors and $1 \%$ of all tumors). Lipomas are commonly found in the subcutaneous tissues (superficial or subcutaneous lipomas) but may extend intramuscularly or along deep fascial planes or beneath enclosing facia, these are known as deep-seated lipomas. Lipoma can be classified based on their anatomical location (superficial and deep), clinical evaluation, or histological [1-4]. Most lipomas occur in the trunk (thorax, abdomen, loin), head, and neck $[1,5]$. Based on the anatomical location there are two types of lipomas: superficial and deep. The superficial lipomas are usually seen in the shoulder, neck, and abdominal region [2, 5]. The deep-seated lipomas occur less frequently than superficial lipomas and may be located above the muscle (supramuscular), between muscle (intermuscular), within the muscle (intramuscular) or below the muscle (submuscular) [3], they can also be found in association with bones (parosteal, and intraosseous), and retroperitoneal region. The sites of involvement of deep-seated lipomas include thorax (anterior chest wall, dorsal and scapular regions, anterior mediastinum), abdomen (anterior abdominal wall, lumbar and loin region, retroperitoneal region), upper limb, lower limb [3, 4]. These tumors are often benign and more 
common in females between 30 and 60 years of age, presenting as a soft circumscribed, movable normally painless mass [3], and the age of onset can vary, they most often develop between the age of 40 and 60 years [6].

Subcutaneous lipomas are the commonest but the deep forms also occur i.e. in 5, 27, 56, 7, and 5\% corresponding to subfascial (sub-aponeurotic or supramuscular), intermuscular, supramuscular, intramuscular, submuscular, and others can be found $[1,4]$. The gross morphology of lipoma manifests as a discrete, mobile, palpable, doughy, solitary soft tissue mass, and can progressively increase in size in asymptomatic fashion. Clinical symptoms are uncommon but they may cause local pain and tenderness, limit the range of motion of a joint or compress the blood supply or nerve to adjoining organs [6]. Superficial lipomas can be accurately diagnosed based on clinical findings in up to $85 \%$ of cases $[7,8]$. Since superficial lipomas are not commonly associated with symptoms except visible swelling, their diagnosis is normally made on a clinical basis, without recourse to radiological workup. A diagnostic test used by some clinicians for superficial lesions is a hardening of the mass after the application of ice [9]. Deep lipomas may also become more apparent as distinct masses during muscle contraction, but their diagnosis usually requires radiological workup. Lipomas may increase in size in periods of fast weight gain, however, it is not true that during periods of severe weight loss lipomas decrease in size, at these times these lesions may become more apparent because the fat in lipomas is unavailable for general metabolism [9]. The most viable treatment option for lipomas is surgical excision [2]. We present a cadaveric case of an intermuscular lipoma seen during routine dissection of cadavers by medical students, we focused on its classification based on anatomical location and histological features, giving their clinical value in a live human context.

The study aimed at evaluating the gross and microscopic features of a deep-seated lipoma and describe it in terms of its clinical anatomy in the context of a live person.

\section{Methods}

\section{Study design}

This was an experimental qualitative and quantitative study conducted to evaluate the gross and microscopic features of a deep-seated lipoma and describe it in terms of its clinical anatomy in the context of a live person. It was conducted at the Laboratory of the Department of Human Anatomy of Kampala International University, Western Campus located in Western Uganda. The samples were sourced from institutional cadavers of Kampala International University, western campus. Our research was conducted in compliance with the Helsinki Declaration with the formal approval of the Scientific and Ethics review committee of Kampala International University Western Campus, Uganda (Nr.UG-REC03/202050). The lipoma specimen used was obtained from a 45-year-old male cadaver during a routine dissection of formalin-fixed adult cadavers with preclinical medical students at Kampala International University, western campus, Uganda. A total of 30 (26 male and 4 female) cadavers were dissected (during the dissection of the back and shoulder region) as part of the medical training in the human 
anatomy course unit. Dissection of the supero-posterior axioappendicular region was performed by a method based on that described by Cunningham based on the usual dissection process [10]. The dissection and observations were carried out by at least three of the authors.

\section{Assessment of the gross and histological features of the lipoma}

The anatomical location of the lipoma was examined in situ, then at the end of the dissection process it was excised from the cadaver for the weight and dimensions to be measured. The tumor weight was measured using a large plastic beaker (BrandTech ${ }^{\circledR}$ ) on an electronic weighing scale (Kent Scientific ${ }^{\circledR}$ ) and this was in line with previous methods $[11,12]$. The morphological dimensions of the mass were assessed using previous methods $[11,12]$. All the measurements were performed thrice using a plastic ruler $(0-30 \mathrm{~cm})$ and recorded in centimeters.

At the end of taking the measurements the fat mass was placed in $10 \%$ formalin solution at room temperature $\left(22-30^{\circ} \mathrm{C}\right)$ for routine histological processing using previous methods [11-13]. Processed lipoma tissues were stained in hematoxylin and eosin and examined under a light microscope (Nikon Eclipse $\mathrm{Ci}, 104 \mathrm{C}$ type) with a mounted digital camera (Nikon digital sight DS, Fi 1) and attached to a computer with software (NIS-Elements F3.00, SP7; Build 547) for photography and data collection. Microscopic analysis was done on $4 \mu \mathrm{m}$ thick paraffin-embedded sections stained in hematoxylin and eosin stain. Tissue was examined in terms of cell type, size, and form, and in terms of arrangement of connective tissue [11-13].

\section{Results}

\section{Gross anatomy findings show an intermuscular lipoma}

The dissection was on the supero-posterior axio-appendicular (supero-posterior chest wall) region of a male 45-year-old cadaver. On gross examination of the mass we saw a well-capsulated homogenous yellow mass was lying between the trapezius muscle above it, and the rhomboid major muscle below (Fig. 1A). Reflection of the tumor revealed that the mass was surrounded by fibrous capsule and firmly adhered with the rhomboid major muscle, also we observed blood vessels piercing through the rhomboid major muscle, then continuing as a prominent branch to invade the tumor through a connective tissue sheath carrying the vessels (Fig. 1B). The fatty tissue nodules were encapsulated by a thin layer of fibrous tissue, and the mass weighed $47.03 \mathrm{~g}$ and measured $8.5 \mathrm{~cm}$ long, $6.6 \mathrm{~cm}$ broad, and $1.7 \mathrm{~cm}$ thick, (Fig. 1C). This is suspected as an intermuscular angiolipoma.

\section{Histopathological Examination Shows Mature Adipose Cells And Angiogenic Invasion}

The histological section of the tissue shows presence of mature adipocytes with prominent blood supply (arterioles and venules). This is diagnosed as an ordinary angiolipoma (Fig. 2). 
Figure 1 here

Figure 2 here

\section{Discussion}

During one of the routine dissection sessions, the students and their supervisors identified a moderately sized mass during dissection of the posterior axio-appendicular region in a male 45-year-old cadaver. This observation became interesting as it provided an avenue for evaluating the gross anatomical and histological aspects of a soft tissue mesenchymal tumor and discuss how it could have affected the living subject. It is the first time we have encountered a lipoma in the course of medical training of students in Gross Anatomy, most medical students may not get to see this on cadavers in their preclinical medical training years. This because the deep-seated lipomas occur less frequently than superficial lipomas [3].

While the etiology of lipomas is unclear, some studies have shown a genetic link, about two-thirds of lipomas demonstrate genetic abnormalities. Apart from the genetic link, one theory claimed a direct positive correlation between trauma to an area and lipoma production. Post-traumatic event following a direct impact on that area of soft tissue is also believed to induce tumors of soft tissues. other possible risk factors that may lead to lipomas are obesity, alcohol abuse, liver disease, as well as, glucose intolerance [3]. Deep lipoma can be classified based on the position of occurrence as subfascial, intramuscular, and intermuscular. The type encountered in this case study was the deep-seated intermuscular type lying under the trapezius and above the rhomboid major muscle getting its blood supply via a branch from the vessel supplying the rhomboid major muscle (dorsal scapular vessel) from where a branch wrapped in connective tissue invaded the lipoma, the invasion of blood vessels was confirmed by histological analysis of fat tissue where blood vessels (venules and arterioles) were identified.

According to anatomical location, our lipoma can be classified as an intermuscular lipoma (between the trapezius and rhomboid major muscles). Intermuscular lipomas are not very common, e.g occur in $27 \%$ of deep lipomas $[4,14]$ and are commonly seen around the anterior abdominal wall, however, ours was found in the supero-posterior axio-appendicular region (dorsal and scapular region of the thorax or posterior chest wall), similar to findings by Paunipagar et al. who found the posterior axioappendicular region of the thorax as the commonest single location (13\%) for the occurrence of the deep-seated lipomas, compared to other locations (anterior abdominal wall, lumbar and loin anterior chest wall, upper and lower limbs [4]. There are few human cases on intermuscular lipomas located between the trapezius and rhomboid muscles (posterior thoracic wall), one typical example is that of a 3-year old male child in Turkey [14], unlike the Turkish case [14] ours was seen in around middle-to-late age (45 years old) as is usually the case with intermuscular lipomas $[4,15]$, however, both the Turkish and the current Ugandan intermuscular lipoma were in the posterior chest wall which is a rare occurrence for them as they are normally found in the anterior abdominal wall $[4,14]$. 
Although lipomas are soft tissue tumors formed by well-demarcated mature adipocytes histologically, they are classified as fibrolipoma, angiolipoma, chondrolipoma, osteolipoma, myxolipoma, myolipoma, and lipoblastoma based on the structures in the masses [2,15]. For instance, it's defined as angiolipoma if the vascular structure is prominent [15]. The type of lipoma seen in this study is having its own blood prominent blood supply and can be described as angiolipoma. Angiogenesis and the production of angiogenic factors are fundamental for tumor progression in the form of growth, invasion, and metastasis $[6,16]$. This particular lipoma would have continued in its growth if not arrested or removed; as a result of the angiogenic connection, it has established with the nearby source of blood supply. Lipomas are common benign tumors of fat cells and mesenchymal tissues. In most cases, surgical excision is curative and simple to perform; however, such a procedure requires general anesthesia and may be associated with delayed wound healing, seroma formation, and nerve injury in deep and intramuscular tumors [17]. Another way of resolving the case is by use of steroid injection via ultrasoundguided intralesional injection of steroid and is found to be partially adequate in most canines [17] especially for the subfascial and subcutaneous growth. Lipoma affects only $1 \%$ of the population, although it is probably under-reported, with higher prevalence in women because females have more subcutaneous fatty tissue than males [18]. However, the lipoma discussed in this case is from a male subject, and we found the lipomas in 1 out of the 30 cadavers dissected (3.3\%) compared to $1 \%$ by Luba et al. [18]. An exception to age rule is the intramuscular lipomas which may occur in all age groups, from childhood to old age, although, the majority of them occur between the ages of 40 and 70 years [19, 20]; however there are contrasting opinions about the occurrence while some studies show a higher occurrence among men between 40-70 [3] others found it is more common in women because of the presence of more subcutaneous fat in this sex $[3,6]$. There is usually no reason for the treatment of most lipomas, as they often pose no threat to the patient, and their growth rate is slow, treatment may be required if a lipoma makes one uncomfortable, by being located on joints (joint and joint capsule lipomas) or if they are rapidly growing to cause impingement of blood vessels and compression of nerves to the adjourning soft tissues, muscles, and joints to cause severe pain and other clinical features in the patient $[9,21]$.

Since the tumor was obtained from a cadaver, we could not establish the history associated with the swelling such as medical, intervention, family, psychosocial and other histories, and follow-up, outcomes, patient perspectives on treatments, and informed consent could not be obtained and therefore not applicable.

\section{Conclusions}

To our knowledge, this is one of the few cases of intermuscular lipomas, especially in East Africa, and is presented and discussed concerning the anatomical location and histological classification. This is a case of intermuscular angiolipoma, the size and the position may cause clinical signs and symptoms of impingement of blood supply and compression of nerves to the adjourning soft tissues, muscles, and joints, hence disfiguring, causing severe pain and other clinical features to the patient if the growth continued undiscovered, unabated and treatment not offered on time. Further studies are recommended 
to establish the aspects of medical, treatment/interventions, family, and psychosocial history, and outcome regarding intermuscular angiolipomas in patients.

\section{Abbreviations}

$\mathrm{H}$ and $\mathrm{E}$ Haematoxylin and Eosin

\section{Declarations}

\section{Ethics approval and consent to participate}

The samples used were from cadavers sourced from institutional cadavers of Kampala International University, western campus, and did not necessitate documented consent for their donation, and informed consent of the patient did not apply since this was a cadaveric case study. Ethical approval was acquired from the Scientific and Ethics review committee of Kampala International University Western Campus, Uganda (Nr.UG-REC-03/202050).

\section{Consent for publication}

Not applicable.

\section{Availability of data and materials}

Not applicable

\section{Competing interests}

The authors declare that they have no competing interests.

\section{Funding}

Not Applicable

\section{Authors' contributions}

A.O.A, O.O.S, A.M.A, F.S conceived the experiments; A.O.A, O.O.S, A.M.A, F.S designed the experiments. A.O.A, O.O.S, A.M.A, F.S, A.D.A acquired the data. A.O.A, O.O.S, A.M.A, F.S, T.P, A.D.A, O.A.O, J.A.T conducted data analysis and interpretation. A.O.A, O.O.S, A.M.A, O.A.O, F.S drafted the initial manuscript. T.P, A.D.A, J.A.T reviewed it for intellectual content while A.O.A, O.O.S, A.M.A, F.S, T.P, A.D.A, O.A.O, J.A.T approved it for publication and remain in agreement to be accountable to all aspects of the work. All the authors have read and agreed to the final manuscript.

\section{Acknowledgments}


We appreciate the Department of Anatomy, Kampala International University, western campus for endorsing the study. We want to express our appreciation to laboratory technicians in the Histopathology laboratory of Kampala International University Teaching Hospital, for their assistance during the processing of the tissue used in this study. Additionally, we appreciate the 200-level medical $\left(1^{\text {st }}\right.$ year preclinical) students at Kampala International University, western campus, Uganda, who were present at the time of finding the mass during a routine dissection session.

\section{References}

1. Kransdorf MJ, Murphey MD. Radiologic Evaluation of Soft-Tissue Masses. Am J Roentgenol. 2000 Sep;175(3):575-87.

2. Rahman G, Abdulkadir A, Yusuf I. Lipomatous lesions around the shoulder: Recent experience in a Nigerian hospital. Int J Shoulder Surg. 2009;3(1):13.

3. McTighe S, Chernev I. Intramuscular lipoma: a review of the literature. Orthop Rev (Pavia). 2014 Dec;16(4):156-63. 6(.

4. Paunipagar BK, Griffith JF, Rasalkar DD, Chow LTC, Kumta SM, Ahuja A. Ultrasound features of deepseated lipomas. Insights Imaging. 2010 Jul 23;1(3):149-53.

5. Murphey M. World Health Organization Classification of Bone and Soft Tissue Tumors: Modifications and Implications for Radiologists. Semin Musculoskelet Radiol. 2007 Sep;11(3):20114.

6. Nishida J, Morita T, Ogose A, Okada K, Kakizaki H, Tajino T, et al. Imaging characteristics of deepseated lipomatous tumors: intramuscular lipoma, intermuscular lipoma, and lipoma-like liposarcoma. J Orthop Sci. 2007 Nov;12(6):533-41.

7. Kleihues P, Eds CWK. World Health Organization classification of tumours. Pathology and genetics of tumours of endocrine organs. 2004.

8. Myhre-Jensen O. A Consecutive 7-Year Series of 1331 Benign Soft Tissue Tumours:

Clinicopathologic Data. Comparison with Sarcomas. Acta Orthop Scand. 1981 Jan;8(3):287-93. 52(.

9. Murphey MD, Carroll JF, Flemming DJ, Pope TL, Gannon FH, Kransdorf MJ. From the Archives of the AFIP. RadioGraphics. 2004 Sep;24(5):1433-66.

10. Koshi R. Cunningham'S Manual of Practical Anatomy Uppwe and Lower Limbs 16Th Edition. 16th ed. Rachel K, editor. Vol. 1. Oxford University Press; 2017. 43-52 p.

11. Heller CA, Marucci DD, Dunn T, Barr EM, Houang M. Dos Remedios C. Inguinal canal ?lipoma? Clin Anat. 2002 Jul;15(4):280-5.

12. Lee YW, Chung J. Intraglandular Ordinary Lipoma of the Submandibular Gland. Ear, Nose Throat J. 2019 Dec 30;014556131989560.

13. Hill C, Flyvbjerg A, Rasch R, Bak M, Logan A. Transforming growth factor- 2 antibody attenuates fibrosis in the experimental diabetic rat kidney. 2001;647-51. 
14. Erikci. A Rare Musculoskeletal Mass: Intermuscular Lipoma I n a Child and Review of Literature. 2019;6(May):6-11.

15. Hagel C, Goebell E, Westphal M, Saeger W. Angiolipoma of the sellar region. Pituitary. 2015 Feb 22;18(1):176-8.

16. Lee JB, Choi HJ, Son ET, Kim JH. A Research of Soft Tissue Lipoma Genesis Factor With Immunohistochemical Analysis. J Craniofac Surg. 2017 Jun;28(4):871-6.

17. Lamagna B, Greco A, Guardascione A, Navas L, Ragozzino M, Paciello O, et al Canine Lipomas Treated with Steroid Injections: Clinical Findings. Debinski W, editor. PLoS One. 2012 Nov 30;7(11):e50234.

18. Luba M, Bangs S, Mohler A, Stulberg D. Approach to Benign Skin Tumors (Papular). Am Fam Physician. 2003;67(4):729-38.

19. Dutton JJ, Wright JD. Intramuscular Lipoma of the Superior Oblique Muscle. Orbit. 2006 Jan 8;25(3):227-33.

20. Fletcher CDM, Martin-Bates E. Intramuscular and intermuscular lipoma: neglected diagnoses. Histopathology. 1988 Mar;12(3):275-87.

21. Bancroft LW, Kransdorf MJ, Peterson JJ, O'Connor MI. Benign fatty tumors: classification, clinical course, imaging appearance, and treatment. Skeletal Radiol. 2006 Oct 1;35(10):719-33.

\section{Figures}



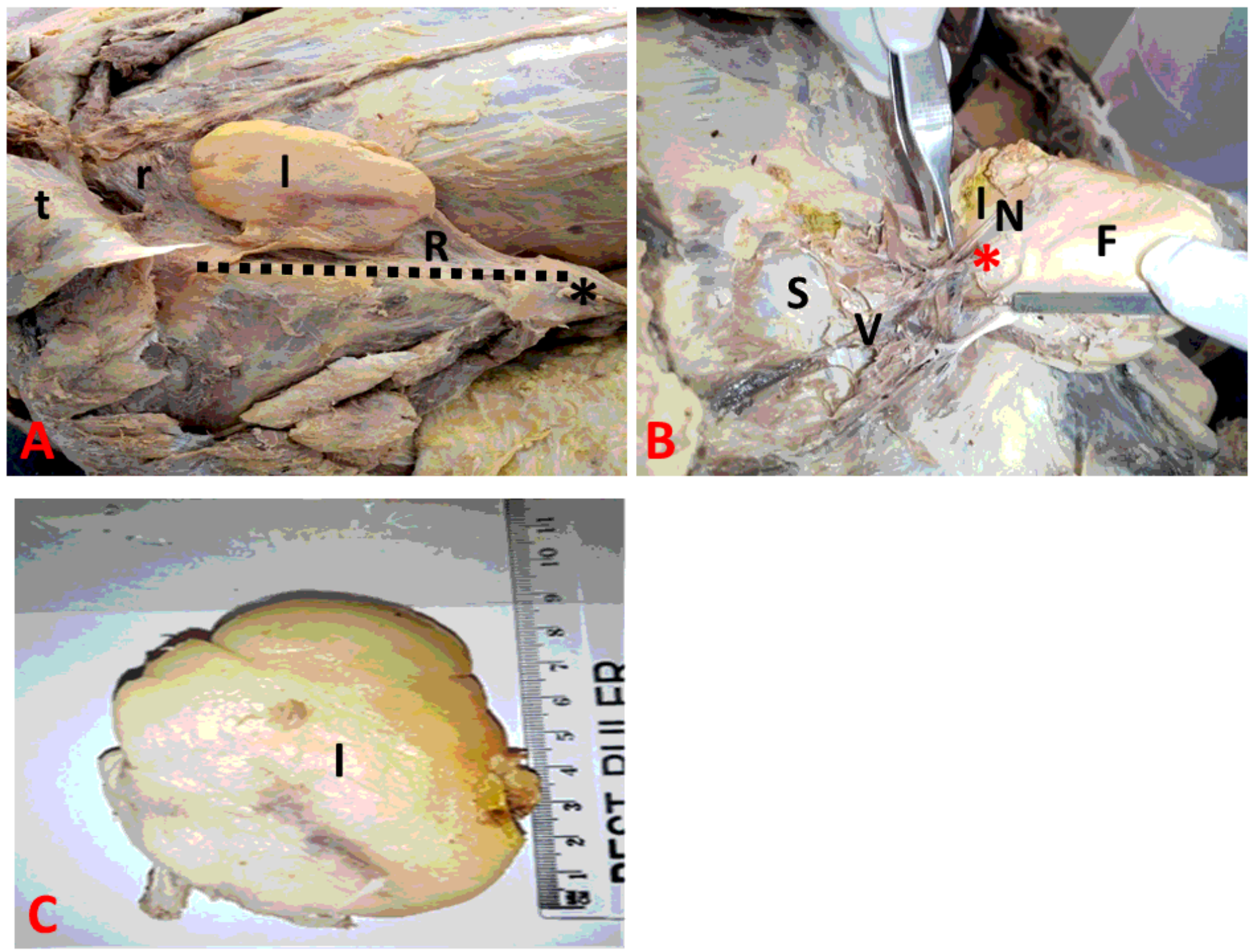

\section{Figure 1}

Photographs showing gross features of a deep intermuscular lipoma in a 45-year adult male cadaver Dorsal view of the dissected supero-posterior axioappendicular region. $A=$ tumor in situ (I) between the reflected trapezius above $(t)$, and rhomboid major muscle beneath $(R) . r=$ rhomboid minor muscle, imaginary features: dotted line $=$ outline of medial border of the scapula, the black star $*=$ inferior angle of the scapula. This is an intermuscular mass. $B=$ reflected both rhomboid major muscle and lipoma tissue (I) showing prominent twig of a blood vessel (red star*) supplying the mass, this is a continuation of the dorsal scapular vessels $(\mathrm{V})$. $\mathrm{F}=$ deep fascia ensheathing rhomboid major muscle, $\mathrm{N}=$ dorsal scapular nerve, $S=$ space normally occupied by rhomboid major muscle before being reflected. Note that the tumor is firmly attached to the muscle. $\mathrm{C}=$ post-dissection excised specimen (I) showing yellow homogenous lobulated appearance (lipoma). 

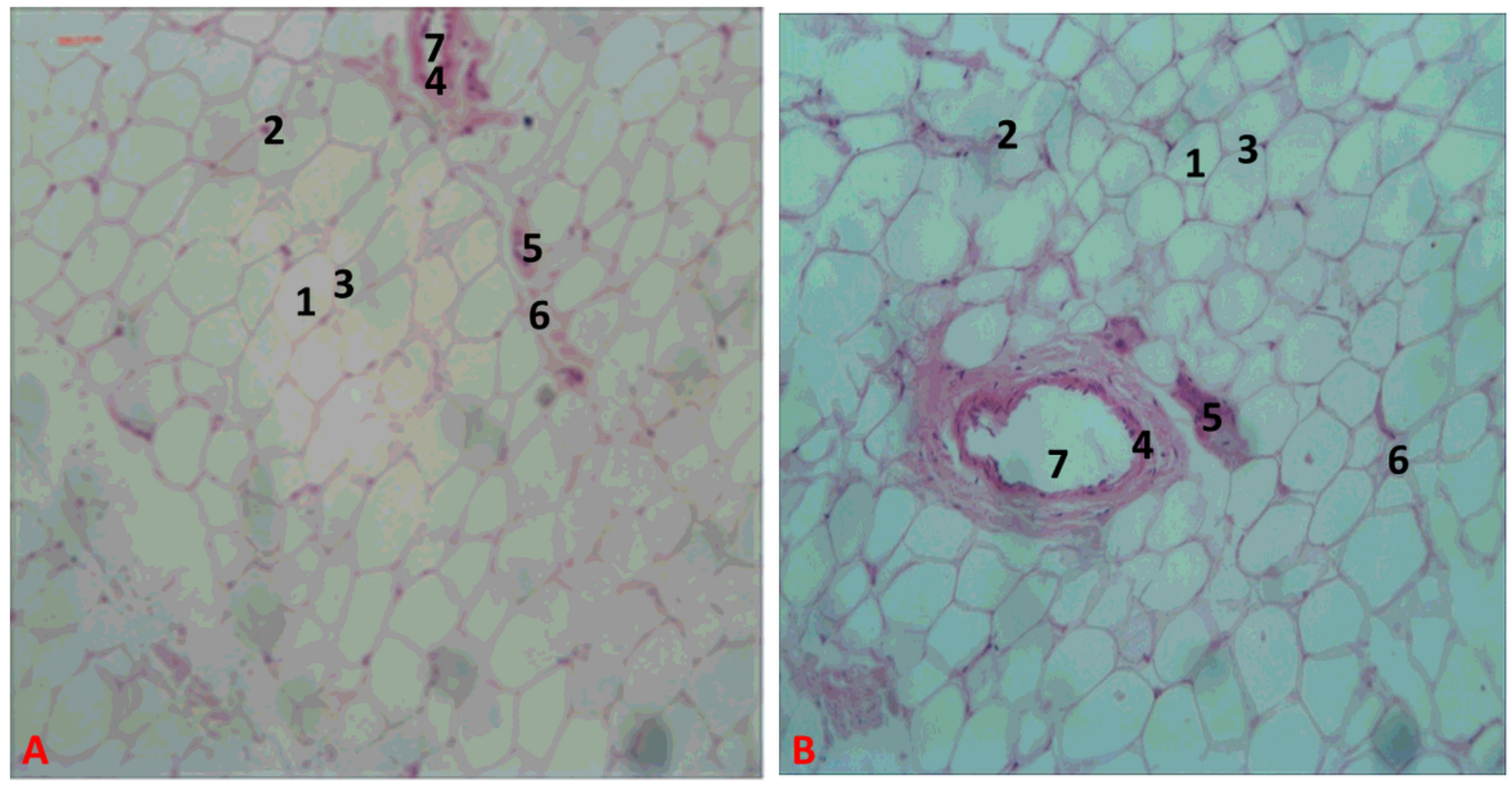

\section{Figure 2}

Microscopic features of a deep intermuscular angiolipoma in a 45-year adult male cadaver $\mathrm{H} \& \mathrm{E}$ photomicrographs (transverse sections). $A=x 40, B=x 100.1=$ fat vacuole, $2=$ adipocyte nucleus, $3=$ adipocyte cytoplasm, $4=$ arteriole, $5=$ venule, $6=$ fibroblasts, $7=$ arteriole lumen. Presence of vascular structures indicates that this is an angiolipoma. 